\title{
Regularity of radial minimizers of reaction equations involving the $p$-Laplacian
}

\author{
Xavier Cabré \\ iCREA And Universitat Politècnica de Catalunya \\ Departament de Matemàtica Aplicada I \\ Diagonal 647, 08028 Barcelona, Spain \\ xavier.cabre@upc.edu \\ Antonio Capella \\ UNIVERSITÄT BONN \\ InStitut Für ANGewANDte Mathematik \\ Wegelerstr. 10, 53115 Bonn, Germany \\ capella@iam.uni-bonn.de \\ MANEL SANChÓN \\ Universitat DE BARCELONA \\ Departament de Matemàtica Aplicada i Anàlisi \\ Gran Via 585, 08007 Barcelona, Spain \\ msanchon@maia.ub.es
}

October 28, 2018

\begin{abstract}
We consider semi-stable, radially symmetric, and decreasing solutions of $-\Delta_{p} u=g(u)$ in the unit ball of $\mathbb{R}^{n}$, where $p>1, \Delta_{p}$ is the $p$-Laplace operator, and $g$ is a locally Lipschitz function. For this class of radial solutions, which includes local minimizers, we establish pointwise, $L^{q}$, and $W^{1, q}$ estimates which are optimal and do not depend on the specific nonlinearity $g$. Among other results, we prove that every radially decreasing and semi-stable solution $u$ belonging to $W^{1, p}\left(B_{1}\right)$ is bounded whenever $n<p+4 p /(p-1)$.

Under standard assumptions on the nonlinearity $g(u)=\lambda f(u)$, where $\lambda>0$ is a parameter, it is proved that the corresponding extremal solution $u^{*}$ is semi-stable, and hence, it enjoys the regularity stated in our main result.
\end{abstract}




\section{Introduction}

This article is concerned with reaction-diffusion equations $-\Delta_{p} u=g(u)$ involving the $p$-Laplace operator. We consider a class of radially symmetric solutions: those solutions which are decreasing and semi-stable. This type of solutions include local minimizers, minimal solutions, extremal solutions, and also certain solutions found between a sub and a supersolution. We establish sharp pointwise, $L^{q}$, and $W^{1, q}$ estimates for solutions in this class. In particular we show that semi-stable radial solutions enjoy better regularity properties than general radial solutions. In addition, our results do not depend on the specific form of the nonlinearity in the reaction term. More precisely, our pointwise and $L^{q}$ estimates hold for every locally Lipschitz nonlinearity $g$, while the $W^{1, q}$ estimates hold for nonnegative $g$. All the results obtained in this paper were obtained by the first two authors in [5] for the Laplace operator, namely $p=2$.

We consider radially symmetric and decreasing solutions $u \in W^{1, p}\left(B_{1}\right)$ of

$$
-\operatorname{div}\left(|\nabla u|^{p-2} \nabla u\right)=g(u) \quad \text { in } B_{1} \backslash\{0\},
$$

where $p>1, B_{1}$ is the unit ball of $\mathbb{R}^{n}$, and $g: \mathbb{R} \longrightarrow \mathbb{R}$ is a locally Lipschitz function. By a radially decreasing function $u$ we mean a function $u$ such that $u=u(r)$ and $u_{r}(r)=(d u / d r)(r)<0$ for all $r \in(0,1)$, where $r=|x|$ and $u_{r}$ denotes the radial derivative.

We do not assume $u(1)=0$ or any other boundary condition. Nevertheless, since $u \in W^{1, p}\left(B_{1}\right)$ is radial, we have $u \in L_{\text {loc }}^{\infty}\left(\bar{B}_{1} \backslash\{0\}\right)$ by the Sobolev embedding in one dimension. Hence, using known regularity results for degenerate elliptic equations (see [21]), we have that in fact $u \in C_{\text {loc }}^{1, \beta}\left(\bar{B}_{1} \backslash\{0\}\right)$ for some $\beta \in$ $(0,1)$. However, $u$ may be unbounded at the origin. Our main result establishes estimates for semi-stable solutions in the whole ball $B_{1}$. Thus, this may be regarded as a result on removable singularities.

Consider the energy functional

$$
E_{\Omega}(u):=\frac{1}{p} \int_{\Omega}|\nabla u|^{p} d x-\int_{\Omega} G(u) d x,
$$

where $G^{\prime}=g$ and $\Omega$ is a smooth bounded domain of $\mathbb{R}^{n}$. We say that a radially decreasing function $u \in W^{1, p}\left(B_{1}\right)$ is a radial local minimizer of (1.2) with $\Omega=B_{1}$ if for every $\delta>0$ there exists $\varepsilon_{\delta}>0$ such that

$$
E_{B_{1} \backslash \bar{B}_{\delta}}(u) \leq E_{B_{1} \backslash \bar{B}_{\delta}}(u+\xi)
$$

for all radial functions $\xi \in C_{c}^{1}\left(B_{1} \backslash \bar{B}_{\delta}\right)$ (i.e., with compact support in $B_{1} \backslash \bar{B}_{\delta}$ ) satisfying $\|\xi\|_{C^{1}} \leq \varepsilon_{\delta}$. Note that every radial local minimizer $u$ is a solution of (1.1). Moreover, $u$ is semi-stable in the sense that the second variation of the energy at $u$ is nonnegative. The following definition makes this precise - see Remark 1.7] for more comments on this, as well as [7] for the corresponding setting in the nonradial case. 
Definition 1.1. Let $u \in W^{1, p}\left(B_{1}\right)$ be a radially symmetric solution in $B_{1} \backslash\{0\}$ of (1.1) such that $u_{r}(r)<0$ for all $r \in(0,1)$. We say that $u$ is semi-stable if and only if the second variation of energy $Q$ at $u$ satisfies

$$
Q(\xi):=\int_{B_{1}}\left\{(p-1)\left|u_{r}\right|^{p-2}\left|\xi_{r}\right|^{2}-g^{\prime}(u) \xi^{2}\right\} d x \geq 0,
$$

for every radially symmetric function $\xi \in C_{c}^{1}\left(B_{1} \backslash\{0\}\right)$.

As we will see later, the class of semi-stable solutions includes not only local minimizers but also the minimal and extremal solutions of the classical problem $\left(1.12_{\lambda, p}\right)$ below, which motivated our work.

To state our estimates, we define exponents $q_{k}$, for $k=0,1$, by

$$
\left\{\begin{array}{lll}
\frac{1}{q_{k}}:=\frac{1}{p}-\frac{2}{n p} \sqrt{\frac{n-1}{p-1}}+\frac{k-1}{n}-\frac{2}{n p} & & \text { for } n \geq p+\frac{4 p}{p-1} \\
q_{k}:=+\infty & & \text { for } n<p+\frac{4 p}{p-1} .
\end{array}\right.
$$

One can easily check that $p<q_{k} \leq+\infty$ in all cases. In addition,

$$
\frac{n-p}{2} \geq 1+\sqrt{\frac{n-1}{p-1}} \quad \text { if and only if } \quad n \geq p+\frac{4 p}{p-1} .
$$

Hence, both exponents $q_{0}$ and $q_{1}$ are well defined. Moreover, $q_{0}$ and $q_{1}$ are finite if $n>p+4 p /(p-1)$.

Our main theorem states sharp regularity results and pointwise estimates for every semi-stable radially decreasing solution $u$ of (1.1). It also establishes estimates for its radial derivative $u_{r}$. Our result extends Theorem 1.5 and part of Theorem 1.8 of [5] (in which $p=2$ ) to the general case $p>1$. In particular, if we set $p=2$ in the following theorem, one recovers the results of [5] for the Laplace operator. The statements in the following theorem are optimal in several respects discussed below, and are still open in general domains even for $p=2$. Indeed, in the nonradial case, they are known to hold only for $g(u)=\lambda e^{u}, g(u)=\lambda(1+u)^{m}$ with $m>p-1$, and nonlinearities $g$ close to them in certain senses - see the comments following Theorem 1.3 for more details.

Theorem 1.2. Let $g$ be a locally Lipschitz function and $u \in W^{1, p}\left(B_{1}\right)$ be a semistable radial solution in $B_{1} \backslash\{0\}$ of (1.1) satisfying $u_{r}(r)<0$ for all $r \in(0,1)$. Then:

(a) If $n<p+4 p /(p-1)$ then $u \in L^{\infty}\left(B_{1}\right)$. Moreover,

$$
\|u\|_{L^{\infty}\left(B_{1}\right)} \leq C_{n, p}\|u\|_{W^{1, p}\left(B_{1}\right)},
$$

where $C_{n, p}$ is a constant depending only on $n$ and $p$. 
(b) If $n=p+4 p /(p-1)$ then $u \in L^{q}\left(B_{1}\right)$ for all $q<+\infty$. Moreover,

$$
|u(r)| \leq C_{p}\|u\|_{W^{1, p}\left(B_{1}\right)}(|\log r|+1) \quad \text { in } B_{1},
$$

where $C_{p}$ is a constant depending only on $p$.

(c) If $n>p+4 p /(p-1)$ and $q<q_{0}$, then $u \in L^{q}\left(B_{1}\right)$ and

$$
\|u\|_{L^{q}\left(B_{1}\right)} \leq C_{n, p, q}\|u\|_{W^{1, p}\left(B_{1}\right)}
$$

where $C_{n, p, q}$ is a constant depending only on $n, p$, and $q$. Moreover,

$$
|u(r)| \leq C_{n, p}\|u\|_{W^{1, p}\left(B_{1}\right)} r^{-\frac{1}{p}\left(n-2 \sqrt{\frac{n-1}{p-1}}-p-2\right)}\left(|\log r|^{\frac{1}{p}}+1\right) \text { in } B_{1}
$$

where $C_{n, p}$ is a constant depending only on $n$ and $p$.

(d) Assume that $g$ is nonnegative. Then:

(d1) We have

$$
\|\nabla u\|_{L^{p}\left(B_{1}\right)} \leq C_{n, p}\left\{\left\|(u-u(1))^{p-1}\right\|_{L^{1}\left(B_{1}\right)}^{\frac{1}{p-1}}+\|g(u)\|_{L^{1}\left(B_{1}\right)}^{\frac{1}{p-1}}\right\}
$$

for some constant $C_{n, p}$ depending only on $n$ and $p$.

(d2) $u \in W^{1, q}\left(B_{1}\right)$ for every $q<q_{1}$, and

$$
\|u\|_{W^{1, q}\left(B_{1}\right)} \leq C \quad \text { if } q<q_{1},
$$

where $C$ is a constant depending only on $n, p, q$, and on upper bounds for $\|u\|_{L^{1}\left(B_{1}\right)}$ and $g$.

(d3) If $n \geq p+4 p /(p-1)$ then

$$
\left|u_{r}(r)\right| \leq C_{n, p}\|u\|_{W^{1, p}\left(B_{1}\right)} r^{-\frac{1}{p}\left(n-2 \sqrt{\frac{n-1}{p-1}}-2\right)}|\log r|^{\frac{1}{p}} \text { in } B_{1 / 4}
$$

where $C_{n, p}$ is a constant depending only on $n$ and $p$.

Examples 1.4 and 1.5 below on the exponential and power nonlinearities show the sharpness of the previous regularity results. Indeed, the functions

$$
u(r)=-p \log r \quad \text { and } \quad u(r)=r^{-\frac{p}{m-(p-1)}}-1
$$

are solutions of (1.1) for, respectively, $g(u)=\lambda^{*} e^{u}$ and $g(u)=\lambda^{*}(1+u)^{m}$ with certain values of $\lambda^{*}=\lambda^{*}(n, p, m)$. Using weighted Hardy inequalities, one can easily find the ranges of values of $n, p$, and $m$ for which these solutions are $W^{1, p}\left(B_{1}\right)$ semi-stable solutions (see [7] and Examples 1.4 and 1.5] below for more details). The ranges obtained in this way for the exponential nonlinearity show the sharpness of condition $n<p+4 p /(p-1)$ in Theorem 1.2(a), as well as the optimality of the pointwise estimate (1.6) in part (b). The ranges obtained for 
the power nonlinearities give the optimality of the exponents $q_{0}$ and $q_{1}$ in parts (c) and (d), as well as the sharpness of the pointwise bounds (1.7) and (1.10) except for the factors $|\log r|^{1 / p}$ in them.

The proof of Theorem 1.2 was inspired in the proof of Simons theorem on the nonexistence of singular minimal cones in $\mathbb{R}^{n}$ for $n \leq 7$ (see [6] for further details). The main point of the proof is to obtain the following key estimate

$$
\int_{B_{1}}\left|u_{r}\right|^{p} r^{-2 \alpha} d x \leq \frac{C_{n, p}}{(n-1)-(\alpha-1)^{2}(p-1)}\|\nabla u\|_{L^{p}\left(B_{1}\right)}^{p}
$$

-from which most of our results follow - for those exponents $\alpha$ making the denominator in the above right-hand side positive (see Lemma 2.3). This estimate is established by taking $\xi=u_{r} \eta$ as a test function on the semi-stability condition (1.3), being $\eta$ essentially a negative power of $r$. Lemma 2.2 shows that, with this choice of $\xi$ in (1.3), the term $g^{\prime}(u)$ in (1.3) disappears. This is the reason why our main estimates do not depend on $g$.

As an application of Theorem 1.2 and the ideas behind its proof, we consider the following problem

$$
\left\{\begin{aligned}
-\operatorname{div}\left(|\nabla u|^{p-2} \nabla u\right) & =\lambda f(u) & & \text { in } B_{1}, \\
u & >0 & & \text { in } B_{1}, \\
u & =0 & & \text { on } \partial B_{1},
\end{aligned}\right.
$$

where $\lambda>0$, and $f$ is an increasing $C^{1}$ function with $f(0)>0$ and

$$
\lim _{t \rightarrow+\infty} \frac{f(t)}{t^{p-1}}=+\infty
$$

Problem $\left(1.12_{\lambda, p}\right)$ is studied in [7] for general smooth bounded domains $\Omega$ of $\mathbb{R}^{n}$. It is proved there that there exists a parameter $\lambda^{*} \in(0, \infty)$ such that if $0<\lambda<\lambda^{*}$ then $\left(1.12_{\lambda, p}\right)$ admits a minimal solution $u_{\lambda} \in C^{1}(\bar{\Omega})$. Here minimal means smaller than any other supersolution of the problem. Moreover, for $\lambda>\lambda^{*}$ problem $\left(1.12_{\lambda, p}\right)$ admits no regular solution. We also have that for every $0<\lambda<\lambda^{*}$ the minimal solution $u_{\lambda}$ is semi-stable (in the sense of Definition 1.1 when $\Omega=B_{1}$ ). Consider the increasing limit

$$
u^{*}:=\lim _{\lambda \uparrow \lambda^{*}} u_{\lambda}
$$

In contrast with the case $p=2$ involving the Laplacian, it is not always clear that the limit $u^{*}$ is a weak solution of $\left(1.12_{\lambda, p}\right)$ for $\lambda=\lambda^{*}$. When one can establish that $u^{*}$ is a weak solution (this may depend on the assumptions on $n, p, \Omega$, and $f)$, it is called the extremal solution.

In our next result we prove that, for $\Omega=B_{1}$, the limit $u^{*}$ is actually a semistable radially decreasing energy solution of $\left(1.12_{\lambda^{*}, p}\right)$. As a consequence, it enjoys the same regularity properties as the ones stated in Theorem 1.2, In particular, 
we obtain that the extremal solution $u^{*}$ is bounded if $n<p+4 p /(p-1)$. As we will see below, the extremal solution is unbounded if $n \geq p+4 p /(p-1)$ when $f(u)=e^{u}$. Therefore, this range of dimensions is optimal. The optimal dimension ensuring the boundedness of the extremal solution in general domains remains unknown for general nonlinearities $f$ under the assumptions above (and even with the additional hypothesis that $f$ is convex).

Theorem 1.3. Let $f$ be a positive and increasing $C^{1}$ function in $[0,+\infty)$ satisfying (1.13). For $\lambda \in\left(0, \lambda^{*}\right)$ let $u_{\lambda}$ be the minimal solution of $\left(1.12_{\lambda, p}\right)$, and let $u^{*}$ be defined by (1.14).

Then,

$$
\left\|u_{\lambda}\right\|_{W^{1, p}\left(B_{1}\right)}+\left\|f\left(u_{\lambda}\right)\right\|_{L^{1}\left(B_{1}\right)} \leq C
$$

for all $\lambda \in\left(0, \lambda^{*}\right)$ and some constant $C$ independent of $\lambda$. Moreover, $u^{*} \in$ $W^{1, p}\left(B_{1}\right)$ and $u^{*}$ is a semi-stable radially decreasing energy solution of $\left(1.12_{\lambda^{*}, p}\right)$. As a consequence, $u^{*}$ has the regularity stated in Theorem 1.2. In particular,

$$
u^{*} \in L^{\infty}\left(B_{1}\right) \quad \text { if } \quad n<p+\frac{4 p}{p-1} .
$$

As mentioned in the statement of the theorem, for problem $\left(1.12_{\lambda, p}\right)$ we consider energy solutions. That is, nonnegative functions $u \in W_{0}^{1, p}\left(B_{1}\right)$ such that $f(u) \in L^{1}\left(B_{1}\right)$ and

$$
\int_{B_{1}}|\nabla u|^{p-2} \nabla u \cdot \nabla \varphi d x=\int_{B_{1}} \lambda f(u) \varphi d x \text { for all } \varphi \in C_{c}^{1}\left(B_{1}\right) .
$$

Note that, by a standard density argument, (1.17) holds for every $\varphi \in W_{0}^{1, p}\left(B_{1}\right)$.

The literature studying minimal and extremal solutions in general domains of $\mathbb{R}^{n}$ is extensive. Crandall and Rabinowitz [8] and Mignot and Puel [22] considered the case of the exponential and power nonlinearities in general domains for $p=2$. They proved that $u^{*}$ is an energy solution for every dimension and that it is bounded in some range of dimensions. These results in general domains were extended for every $p>1$ by García-Azorero, Peral, and Puel [17, 18] for the exponential case, and by Ferrero [16] and Cabré and Sanchón [7] for power type nonlinearities.

An optimal regularity result for the extremal solution is not known for general nonlinearities and general domains, even for $p=2$, with the exception of the radial case $\Omega=B_{1}$. In [5], Cabré and Capella obtained the optimal regularity of the extremal solution for every locally Lipschitz nonlinearity $f$ when the domain is a ball and $p=2$. In particular, they proved that the extremal solution is bounded if $n \leq 9$. In this paper, we obtain an analogue optimal radial result for all $p>1$.

In the nonradial case, [23] and [2] contain the best results for $p=2$. In [23], Nedev proves for $p=2$ that the extremal solution is bounded if $n \leq 3$-i.e., 
$n<4$ in (1.18) - whenever $f$ is an increasing and convex function such that $f(0)>0$ and (1.13) holds. The same result up to dimension $n \leq 4$ is proved by the first author in [2] for $p=2$ and $\Omega$ convex, without the convexity assumption on $f$. On the other hand, the third author extends in [27] the work of Nedev [23] to the case $p>2$ and establishes

$$
u^{*} \in L^{\infty}(\Omega) \quad \text { if } \quad n<p+\frac{p}{p-1} .
$$

Note that for $p=2$, we recover Nedev's condition $n<4$. Note also the existing gap between the dimension in (1.18) for general $\Omega$ and the ones in our result for $\Omega=B_{1}$-note the presence of the factor 4 in (1.16) in contrast with (1.18).

We refer to [10, 3] for surveys on minimal and extremal solutions and to [1, 11, 12, 13, 14, 15, 19, 20, 26] for other interesting results in the topic of extremal solutions - also for problems involving other operators, such as the bilaplacian, or fractional Laplacians related to boundary reactions.

The key point in the proof of Theorem 1.3 is to establish the uniform estimate (1.15) on the $W^{1, p}$-norm and the $L^{1}\left(B_{1}\right)$-norm of $u_{\lambda}$ and $f\left(u_{\lambda}\right)$, respectively. This is accomplished using the "superlinearity" hypothesis (1.13) on $f$, together with the radially decreasing character of the solutions. The $W^{1, p}$ bound is then used to show that the limit $u^{*}$ is an energy solution of $\left(1.12_{\lambda^{*}, p}\right)$. Using again that the $u_{\lambda}$ are semi-stable, we prove that $u^{*}$ is semi-stable.

In [7, the semi-stability property of the extremal solution $u^{*}$ was proved in the case $p \geq 2$ for general domains in $\mathbb{R}^{n}$ and nonlinearities having its growth comparable to a power. This property was obtained taking the limit as $\lambda \rightarrow \lambda^{*}$ in the semi-stability condition for the minimal solutions $u_{\lambda}$. In the case $1<p<2$ this argument does not apply for general domains, since we have no control on the set where the gradient $\nabla u^{*}$ vanishes. However, in the radial case this set is the origin, and thus we obtain here the result of [7] also for $1<p<2$.

Next, we introduce two explicit examples which show the optimality of Theorems 1.2 and 1.3 .

Example 1.4. In [17, 18] García-Azorero, Peral, and Puel considered problem $\left(1.12_{\lambda, p}\right)$ for $f(u)=e^{u}$ and a general bounded domain $\Omega$. They proved that if $n<p+4 p /(p-1)$ then the extremal solution $u^{*}$ is bounded. On the other hand, if $n \geq p+4 p /(p-1)$ and $\Omega=B_{1}$ they show that

$$
u^{*}(r)=-p \log r \quad \text { and } \quad \lambda^{*}=p^{p-1}(n-p),
$$

and hence, that the extremal solution is unbounded. This example shows that the conditions on the dimension $n$ in terms of $p$ in Theorem 1.2(a),(b) and the pointwise estimate (1.6) are optimal.

Indeed, as mentioned before, it is easy to check that for the previous range of dimensions, $u^{*}$ as in (1.19) is a singular $W^{1, p}$ semi-stable solution -independently of the more precise fact of actually being the extremal solution. This remark also applies to the following example. 
Example 1.5. Consider the power nonlinearity $f(u)=(1+u)^{m}$, with $m>p-1$, which is studied in [7] and [16] for general domains. Let us define the following critical exponent

$$
m_{c s}(p):= \begin{cases}\frac{(p-1) n-2 \sqrt{(p-1)(n-1)}+2-p}{n-(p+2)-2 \sqrt{\frac{n-1}{p-1}}} & \text { if } n>p+\frac{4 p}{p-1} \\ +\infty & \text { if } n \leq p+\frac{4 p}{p-1}\end{cases}
$$

The results of [7, 16] show that if $m<m_{c s}(p)$ then the extremal solution $u^{*}$ of $\left(1.12_{\lambda, p}\right)$ is bounded. On the other hand, if $\Omega=B_{1}$ and $m \geq m_{c s}(p)$ then

$$
u^{*}(r)=r^{\frac{-p}{m-(p-1)}}-1 \quad \text { and } \quad \lambda^{*}=\left(\frac{p}{m-(p-1)}\right)^{p-1}\left(n-\frac{m p}{m-(p-1)}\right) .
$$

We see that $u^{*} \in L^{q}\left(B_{1}\right)$ if and only if $1 \leq q<n(m-(p-1)) / p$. Now, if we let $m=m_{c s}(n, p)$ then we find that $n\left(m_{c s}(n, p)-(p-1)\right) / p=q_{0}$ and this proves the sharpness of Theorem $1.2(\mathrm{c})$.

Observe that in the same case $m=m_{c s}(n, p)$ we have

$$
u^{*}(r)=r^{-\frac{1}{p}\left(n-2 \sqrt{\frac{n-1}{p-1}}-p-2\right)}-1,
$$

and, as in the case $p=2$, this differs from the pointwise bound (1.7) for the factor $|\log r|^{1 / p}$. Recently, Villegas [29] has proved that, for $p=2$, the factor $|\log r|^{1 / 2}$ in (1.7) can be removed. See also [28] for a related improvement of a radial Liouville theorem of [4].

Remark 1.6. The critical dimension for the boundedness of semi-stable solutions determined by $n=p+4 p /(p-1)$ tends to $+\infty$ as $p \rightarrow 1$ and as $p \rightarrow+\infty$. The smallest critical dimension corresponds to $p=3$, for which $n=9$. In the cases $p=2$ and $p=5$ we find the value $n=10$. Observe that case (b) in Theorem 1.3, $n=p+4 p /(p-1)$, applies only for those $p>1$ such that $p+4 p /(p-1)$ is an integer. Note also that the critical dimension for the boundedness of all semistable solutions is bigger than the corresponding dimension for general solutions, which is $n<p$. Therefore, semi-stable solutions enjoy more regularity than general solutions.

Remark 1.7. The following two comments concern the notion of semi-stability of solutions. See [7] for a more general nonradial setting.

(i) If $u$ is a radially decreasing local minimizer as defined after (1.2), we claim that $u$ is a semi-stable solution. Therefore, Theorem 1.2 applies to every radially decreasing local minimizer $u \in W^{1, p}\left(B_{1}\right)$. To check this, note that the second 
variation of the energy functional (1.2) in $B_{1}$ at a radially decreasing solution $u$ is given by

$$
\int_{B_{1}}\left\{|\nabla u|^{p-2}\left((p-2)\left[\frac{\nabla u}{|\nabla u|} \cdot \nabla \xi\right]^{2}+|\nabla \xi|^{2}\right)-g^{\prime}(u) \xi^{2}\right\} d x
$$

for every perturbation $\xi \in C^{1}$ with compact support in $B_{1} \backslash\{0\}$ (not necessarily radially symmetric). It follows that, if $u$ is a radial local minimizer, then (1.20) is nonnegative for every radial $\xi \in C_{c}^{1}\left(B_{1} \backslash\{0\}\right)$. Therefore, $u$ is semi-stable in the sense of Definition 1.1 since for radial perturbations $\xi$, (1.20) reduces to (1.3).

(ii) If $u$ is a radially decreasing semi-stable solution of (1.1) in the sense of Definition 1.1, we claim that $(1.20)$ is nonnegative for every nonradial $\xi \in C^{1}$ with compact support in $B_{1} \backslash\{0\}$. That is, the second variation of energy at $u$ is nonnegative not only for radial perturbations but also for nonradial ones. Indeed, for $x \in \mathbb{R}^{n}$ we set $x=r \theta$, where $r=|x| \geq 0$, and $\theta=x / r \in \partial B_{1}=\{\theta \in$ $\left.\mathbb{R}^{n}:|\theta|=1\right\}$. Let $\xi \in C_{c}^{1}\left(B_{1} \backslash\{0\}\right.$ ) (not necessarily radial). We consider the spherical averages of $\xi^{2}$ and define the following radial function:

$$
\varphi^{2}(r):=\frac{1}{\left|\partial B_{1}\right|} \int_{\partial B_{1}} \xi^{2}(r \theta) d \theta=\int_{\partial B_{1}} \xi^{2}(r \theta) d \theta .
$$

Differentiating the last expression with respect to $r$ and using the Cauchy-Schwarz inequality, we find

$$
\varphi_{r}^{2}(r) \leq \int_{\partial B_{1}}\left[\frac{x}{r} \cdot \nabla \xi(r \theta)\right]^{2} d \theta=\oint_{\partial B_{1}}\left|\xi_{r}(r \theta)\right|^{2} d \theta
$$

Finally, after some straightforward computations, and using the semi-stability of $u$ in the sense of Definition 1.1 for the radial test function $\varphi=\varphi(r)$ and that $\xi_{r}^{2} \leq|\nabla \xi|^{2}$ in (1.20), the claim follows.

The paper is organized as follows. In Section 2 we prove the main estimates on semi-stable stable solutions needed in the rest of the paper. In Section 3 we prove Theorem [1.2, while Section 4 is devoted to prove Theorem 1.3 .

\section{Estimates for semi-stable solutions}

This section is devoted to show Lemma 2.3, which contains the key estimate used in the proof of Theorem 1.2 .

We begin with the following remark on radial functions and radially decreasing solutions of (1.11). 
Remark 2.1. Given $\delta \in(0,1)$, every radial function in $W^{1, p}\left(B_{1}\right)$ also belongs (as a function of $r=|x|$ ) to the Sobolev space $W^{1, p}(\delta, 1)$ in one dimension. As a consequence, by the Sobolev embedding in one dimension, $u$ is a continuous function of $r \in[\delta, 1]$,

$$
|u(1)| \leq C_{n, p}\|u\|_{W^{1, p}\left(B_{1}\right)} \quad \text { and } \quad\|u\|_{L^{\infty}\left(B_{1} \backslash \bar{B}_{\delta}\right)} \leq C_{n, p, \delta}\|u\|_{W^{1, p}\left(B_{1}\right)}
$$

for some constant $C_{n, p}$ (respectively, $C_{n, p, \delta}$ ) depending only on $n$ and $p$ (respectively, on $n, p$, and $\delta$ ).

Now, let $u \in W^{1, p}\left(B_{1}\right)$ be a radial solution to (1.1) such that $u_{r}(r)<0$ for all $r \in(0,1)$. By (2.1), the right-hand side of (1.1) is bounded away from the origin. Hence, by standard regularity theory for the $p$-Laplacian, $u \in C_{\text {loc }}^{1, \beta}\left(\bar{B}_{1} \backslash\{0\}\right)$ for some $\beta \in(0,1)$. In particular, $u_{r}$ is a continuous function in $B_{1} \backslash\{0\}$ which does not vanish and thus (1.1) is uniformly elliptic in compact sets of $B_{1} \backslash\{0\}$. It follows that $u \in C_{\text {loc }}^{2, \beta}\left(B_{1} \backslash\{0\}\right)$.

Since $u_{r}<0$ in $B_{1} \backslash\{0\}$, we can write (1.1) in radial coordinates as

$$
-r^{1-n} \partial_{r}\left(r^{n-1}\left|u_{r}\right|^{p-2} u_{r}\right)=g(u) \quad \text { for } r \in(0,1),
$$

and also in the form

$$
\text { - }(p-1)\left|u_{r}\right|^{p-2} \partial_{r} u_{r}-\frac{n-1}{r}\left|u_{r}\right|^{p-2} u_{r}=g(u) \quad \text { for } r \in(0,1) .
$$

In order to show our main estimate (stated in Lemma 2.3) we need a preliminary result on the form of the second variation of the energy for radial solutions. This lemma was inspired in the proof of Simons theorem on the nonexistence of singular minimal cones in $\mathbb{R}^{n}$ for $n \leq 7$ (see [4, 6] for further details).

Lemma 2.2. Let $u \in W^{1, p}\left(B_{1}\right)$ be a radial solution in $B_{1} \backslash\{0\}$ of (1.1) satisfying $u_{r}(r)<0$ for all $r \in(0,1)$ and $Q$ be the quadratic form defined in (1.3). Then,

$$
Q\left(u_{r} \eta\right)=\int_{B_{1}}\left|u_{r}\right|^{p}\left\{(p-1)\left|\eta_{r}\right|^{2}-\frac{n-1}{r^{2}} \eta^{2}\right\} d x,
$$

for every radial function $\eta \in C_{c}^{1}\left(B_{1} \backslash\{0\}\right)$, that is, with compact support in $B_{1} \backslash\{0\}$.

Note that expression (2.4) for the quadratic form $Q$ does not contain any reference to the nonlinearity $g$. This is the reason why our estimates do not depend on the nonlinearity $g$.

Proof of Lemma 2.2. By Remark 2.1, $u \in C_{\mathrm{loc}}^{2, \beta}\left(B_{1} \backslash\{0\}\right)$ for some $\beta \in(0,1)$ and $u$ satisfies equation (2.3). Let us set $H(u)=(p-1)\left|u_{r}\right|^{p-2}$. Let $\eta \in C_{c}^{1}\left(B_{1} \backslash\{0\}\right)$ 
be a radial function with compact support in $B_{1} \backslash\{0\}$ and $c \in C^{1}\left(B_{1} \backslash\{0\}\right)$ be a radial function. Take $\xi=c \eta \in C_{c}^{1}\left(B_{1} \backslash\{0\}\right)$ in (1.3) to obtain

$$
\begin{aligned}
Q(c \eta) & =\int_{B_{1}}\left\{H(u)|\nabla(c \eta)|^{2}-g^{\prime}(u) c^{2} \eta^{2}\right\} d x \\
& =\int_{B_{1}}\left\{H(u)\left(c^{2}|\nabla \eta|^{2}+\nabla \eta^{2} \cdot c \nabla c+\eta^{2}|\nabla c|^{2}\right)-g^{\prime}(u) c^{2} \eta^{2}\right\} d x \\
& =\int_{B_{1}}\left\{H(u) c^{2}|\nabla \eta|^{2}+H(u) \nabla\left(\eta^{2} c\right) \cdot \nabla c-g^{\prime}(u) c^{2} \eta^{2}\right\} d x .
\end{aligned}
$$

Next, we multiply (2.3) by $\partial_{r}\left(\eta^{2} u_{r} r^{n-1}\right)$, we integrate in $r$ from 0 to 1 and use integration by parts to obtain

$$
0=\int_{0}^{1} \partial_{r}\left(\eta^{2} u_{r} r^{n-1}\right) H(u) \partial_{r} u_{r} d r-\int_{0}^{1} \partial_{r}\left\{\frac{n-1}{r}\left|u_{r}\right|^{p-2} u_{r}+g(u)\right\} \eta^{2} u_{r} r^{n-1} d r .
$$

Using $\partial_{r}\left(\left|u_{r}\right|^{p-2} u_{r}\right)=H(u) u_{r r}$ and $r^{n-1} d r=\left|\partial B_{1}\right|^{-1} d x$, we deduce

$$
0=\int_{B_{1}} H(u) \partial_{r}\left(\eta^{2} u_{r}\right) \partial_{r} u_{r} d x-\int_{B_{1}}\left\{g^{\prime}(u) \eta^{2} u_{r}^{2}-\frac{n-1}{r^{2}}\left|u_{r}\right|^{p-2} u_{r}^{2} \eta^{2}\right\} d x
$$

Therefore

$$
\int_{B_{1}}\left\{H(u) \nabla\left(\eta^{2} u_{r}\right) \cdot \nabla u_{r}-g^{\prime}(u) \eta^{2} u_{r}^{2}\right\} d x=-\int_{B_{1}} \frac{n-1}{r^{2}} \eta^{2}\left|u_{r}\right|^{p} d x .
$$

Taking $c=u_{r}$ in (2.5) and using (2.6), we obtain (2.4).

Now, we use Lemma 2.2 and the semi-stability assumption to establish the following result. It is an $L^{p}$ estimate for $u_{r} r^{-2 \alpha / p}$ in $B_{1}$, for certain positive exponent $\alpha$ depending on $n$ and $p$, in terms of the $W^{1, p}$ norm of $u$. As said before, this is the key estimate in the proof of Theorem 1.2. Here as in the rest of this section, we assume that $n \geq p$. Note that when $n<p$, we have $W^{1, p}\left(B_{1}\right) \subset L^{\infty}\left(B_{1}\right)$ and hence solutions are bounded.

Lemma 2.3. Assume $n \geq p$. Let $u \in W^{1, p}\left(B_{1}\right)$ be a semi-stable radial solution in $B_{1} \backslash\{0\}$ of (1.1) satisfying $u_{r}(r)<0$ for $r \in(0,1)$. Let $\alpha$ satisfy

$$
1 \leq \alpha<1+\sqrt{\frac{n-1}{p-1}}
$$

Then

$$
\int_{B_{1}}\left|u_{r}\right|^{p} r^{-2 \alpha} d x \leq \frac{C_{n, p}}{(n-1)-(\alpha-1)^{2}(p-1)}\|\nabla u\|_{L^{p}\left(B_{1}\right)}^{p},
$$

where $C_{n, p}$ is a constant depending only on $n$ and $p$. 
Proof. By the semi-stability of $u$ and Lemma 2.2 applied with $\eta$ replaced by $r \eta$, we have that

$$
(n-1) \int_{B_{1}}\left|u_{r}\right|^{p} \eta^{2} d x \leq(p-1) \int_{B_{1}}\left|u_{r}\right|^{p}|\nabla(r \eta)|^{2} d x
$$

holds for every radial $\eta \in C_{c}^{1}\left(B_{1} \backslash\{0\}\right)$. Since $\eta$ vanishes in a neighborhood of the origin and $u \in C^{1}$ away from the origin (see Remark 2.1), we deduce by an approximation argument that (2.9) also holds for every radial Lipschitz function $\eta$ vanishing on $\partial B_{1}$ and also in a neighborhood of the origin.

We now prove that (2.9) also holds for every radial Lipschitz function vanishing on $\partial B_{1}$ - but now not necessarily vanishing around 0 . To see this, take $\zeta \in C^{1}\left(\mathbb{R}^{n}\right)$ such that $0 \leq \zeta \leq 1, \zeta \equiv 0$ in $B_{1}$, and $\zeta \equiv 1$ in $\mathbb{R}^{n} \backslash B_{2}$. Let $\zeta_{\delta}(\cdot):=\zeta(\cdot / \delta)$ for every $\delta>0$. Replacing $\eta$ by $\eta \zeta_{\delta}$ (which is Lipschitz and vanishes in a neighborhood of $\{0\})$ in (2.9), we obtain

$$
(n-1) \int_{B_{1}}\left|u_{r}\right|^{p} \zeta_{\delta}^{2} \eta^{2} d x \leq(p-1) \int_{B_{1}}\left|u_{r}\right|^{p}\left|\nabla\left(r \eta \zeta_{\delta}\right)\right|^{2} d x .
$$

Now, using that $\eta$ and $\nabla \eta$ are bounded and denoting $A_{\delta, 2 \delta}=B_{2 \delta} \backslash \bar{B}_{\delta}$ we find

$$
\begin{aligned}
\int_{B_{1}}\left|u_{r}\right|^{p}\left|\nabla\left(r \eta \zeta_{\delta}\right)\right|^{2} d x & \\
& =\int_{B_{1}}\left|u_{r}\right|^{p}\left\{|\nabla(r \eta)|^{2} \zeta_{\delta}^{2}+r^{2} \eta^{2}\left|\nabla \zeta_{\delta}\right|^{2}+\zeta_{\delta} \nabla \zeta_{\delta} \cdot \nabla\left(r^{2} \eta^{2}\right)\right\} d x \\
& \leq \int_{B_{1}}\left|u_{r}\right|^{p}|\nabla(r \eta)|^{2} \zeta_{\delta}^{2} d x+C \int_{A_{\delta, 2 \delta}}\left|u_{r}\right|^{p}|\eta|\left\{\frac{r^{2}}{\delta^{2}}|\eta|+\frac{r}{\delta}\left|\zeta_{\delta}\right||\nabla(r \eta)|\right\} d x \\
& \leq \int_{B_{1}}\left|u_{r}\right|^{p}|\nabla(r \eta)|^{2} \zeta_{\delta}^{2} d x+C \int_{A_{\delta, 2 \delta}}\left|u_{r}\right|^{p} d x
\end{aligned}
$$

where $C$ denotes different positive constants. Since $u \in W^{1, p}\left(B_{1}\right)$, the last term in (2.11) tends to zero as $\delta$ goes to zero. Therefore using that $\zeta_{\delta}$ tend to 1 a.e. in $B_{1}$ as $\delta \rightarrow 0$, (2.11), and (2.10), we obtain by monotone convergence that (2.9) holds for every radial Lipschitz function $\eta$ vanishing on $\partial B_{1}$.

For $\alpha$ satisfying (2.7) and $\varepsilon \in(0,1)$, let

$$
\eta_{\varepsilon}(r):= \begin{cases}\varepsilon^{-\alpha}-1 & \text { for } 0 \leq r \leq \varepsilon \\ r^{-\alpha}-1 & \text { for } \varepsilon<r \leq 1\end{cases}
$$

a Lipschitz function vanishing on $\partial B_{1}$. From inequality (2.9) applied with $\eta=\eta_{\varepsilon}$, we obtain

$$
\begin{aligned}
& (n-1) \int_{B_{1} \backslash B_{\varepsilon}}\left|u_{r}\right|^{p}\left(r^{-\alpha}-1\right)^{2} d x+(n-1)\left(\varepsilon^{-\alpha}-1\right)^{2} \int_{B_{\varepsilon}}\left|u_{r}\right|^{p} d x \\
& \leq(p-1) \int_{B_{1} \backslash B_{\varepsilon}}\left|u_{r}\right|^{p}\left\{(1-\alpha) r^{-\alpha}-1\right\}^{2} d x+(p-1)\left(\varepsilon^{-\alpha}-1\right)^{2} \int_{B_{\varepsilon}}\left|u_{r}\right|^{p} d x .
\end{aligned}
$$


Since $n \geq p$ it follows that

$$
(n-1) \int_{B_{1} \backslash B_{\varepsilon}}\left|u_{r}\right|^{p}\left(r^{-\alpha}-1\right)^{2} d x \leq(p-1) \int_{B_{1} \backslash B_{\varepsilon}}\left|u_{r}\right|^{p}\left\{(1-\alpha) r^{-\alpha}-1\right\}^{2} d x .
$$

Developing the squares, using $n \geq p$ and (2.7), we find the estimate

$$
\int_{B_{1} \backslash B_{\varepsilon}}\left|u_{r}\right|^{p} r^{-2 \alpha} d x \leq \frac{C_{n, p}}{(n-1)-(\alpha-1)^{2}(p-1)} \int_{B_{1} \backslash B_{\varepsilon}}\left|u_{r}\right|^{p} r^{-\alpha} d x .
$$

Throughout the proof $C_{n, p}$ (respectively $C_{n, p, \alpha}$ ) denote different positive constants depending only on $n$ and $p$ (respectively on $n, p$, and $\alpha$ ).

Now, choose a positive constant $C_{n, p, \alpha}$ such that

$$
\frac{C_{n, p}}{(n-1)-(\alpha-1)^{2}(p-1)} r^{-\alpha} \leq \frac{1}{2} r^{-2 \alpha}+C_{n, p, \alpha} \quad \text { for all } r \in(0,1) .
$$

Combining this inequality and (2.12), we obtain

$$
\int_{B_{1} \backslash B_{\varepsilon}}\left|u_{r}\right|^{p} r^{-2 \alpha} d x \leq C_{n, p, \alpha} \int_{B_{1} \backslash B_{\varepsilon}}\left|u_{r}\right|^{p} d x .
$$

Now, let $\varepsilon \rightarrow 0$ to conclude

$$
\int_{B_{1}}\left|u_{r}\right|^{p} r^{-2 \alpha} d x \leq C_{n, p, \alpha}\|\nabla u\|_{L^{p}\left(B_{1}\right)}^{p}
$$

In order to find a more precise expression on how the previous constant $C_{n, p, \alpha}$ depends on $\alpha$, we apply (2.13) with the especial choice $\alpha=\alpha_{0}$ given by

$$
\alpha_{0}=\frac{1}{2}+\frac{1}{2} \sqrt{\frac{n-1}{p-1}} \in\left[1,1+\sqrt{\frac{n-1}{p-1}}\right) .
$$

We deduce

$$
\int_{B_{1}}\left|u_{r}\right|^{p} r^{-1-\sqrt{\frac{n-1}{p-1}}} d x \leq C_{n, p}\|\nabla u\|_{L^{p}\left(B_{1}\right)}^{p} .
$$

Finally, since $r^{-\alpha} \leq r^{-(1+\sqrt{(n-1) /(p-1)})}$ in $B_{1}$, (2.12) and (2.14) lead to the desired estimate (2.8) after letting $\varepsilon \rightarrow 0$.

\section{Pointwise, $L^{q}$, and $W^{1, q}$ estimates}

In this section we prove Theorem 1.2 . 
Proof of Theorem 1.2. By (2.1), $|u(1)| \leq C_{n, p}\|u\|_{W^{1, p}\left(B_{1}\right)}$. Hence, in view of the estimates that we need to prove, it suffices to establish them with $u$ replaced by $u-u(1)-$ a positive solution vanishing on $\partial B_{1}$. Thus, through the proof, we assume

$$
u>0=u(1) \text { in } B_{1} .
$$

In case $n<p$, since $u \in W^{1, p}\left(B_{1}\right)$, the Sobolev embedding leads to $u \in$ $L^{\infty}\left(B_{1}\right)$ and $\|u\|_{L^{\infty}\left(B_{1}\right)} \leq C_{n, p}\|u\|_{W^{1, p}\left(B_{1}\right)}$.

In case $n \geq p$, let $\alpha$ satisfy (2.7). For $0<t<1$ we have

$$
\begin{aligned}
u(t) & =\int_{t}^{1}-u_{r} r^{-(2 \alpha-n+1) / p} r^{(2 \alpha-n+1) / p} d r \\
& \leq C_{n, p}\left(\int_{B_{1}}\left|u_{r}\right|^{p} r^{-2 \alpha} d x\right)^{\frac{1}{p}}\left(\int_{t}^{1} r^{p^{\prime}(2 \alpha-n+1) / p} d r\right)^{\frac{1}{p^{\prime}}},
\end{aligned}
$$

by Hölder inequality and where $p^{\prime}=p /(p-1)$. Using Lemma 2.3 (which requires $n \geq p)$ we deduce

$$
u(t) \leq \frac{C_{n, p}\|u\|_{W^{1, p}\left(B_{1}\right)}}{\left\{(n-1)-(\alpha-1)^{2}(p-1)\right\}^{\frac{1}{p}}}\left(\int_{t}^{1} r^{p^{\prime}(2 \alpha-n+1) / p} d r\right)^{\frac{1}{p^{\prime}}}
$$

for all $0<t<1$.

(a) Assume $n<p+4 p /(p-1)$. By the remark made above, we may assume that $n \geq p$. Note that the integral in (3.2) is finite with $t=0$ whenever $p^{\prime}(2 \alpha-$ $n+1) / p>-1$, or equivalently

$$
\int_{0}^{1} r^{p^{\prime}(2 \alpha-n+1) / p} d r<+\infty \quad \text { if } \frac{n-p}{2}<\alpha .
$$

Recalling (1.5) and since $n<p+4 p /(p-1)$, we can choose $\alpha$ (depending only on $n$ and $p$ ) satisfying

$$
\frac{n-p}{2}<\alpha<1+\sqrt{\frac{n-1}{p-1}} .
$$

In addition, we may take $\alpha \geq 1$-as required in (2.7). Now, the desired $L^{\infty}$ estimate follows from (3.2) and (3.3).

(b) Assume $n=p+4 p /(p-1)$. Let $\varepsilon \in(0,1)$ and

$$
\alpha=1+\sqrt{\frac{n-1}{p-1}}-\varepsilon=\frac{2 p}{p-1}-\varepsilon .
$$

Since $\alpha$ satisfies (2.7) in Lemma 2.3, (3.2) yields

$$
\begin{aligned}
u(t) & \leq \frac{C_{p}\|u\|_{W^{1, p}\left(B_{1}\right)}}{\varepsilon^{\frac{1}{p}}}\left(\int_{t}^{1} r^{-1-2 \varepsilon p^{\prime} / p} d r\right)^{\frac{1}{p^{\prime}}} \\
& \leq \frac{C_{p}\|u\|_{W^{1, p}\left(B_{1}\right)}}{\varepsilon} t^{-2 \varepsilon / p}
\end{aligned}
$$


for all $0<t<1$ and $0<\varepsilon<1$, where $C_{p}=C_{p, n}$ is a constant depending only in $p$ (since here $n$ is a function of $p$ ). It follows that $u \in L^{q}\left(B_{1}\right)$ for every $1 \leq q<\infty$.

In order to prove the pointwise estimate (1.6), we optimize the right-hand side of (3.4) with respect to $\varepsilon$ by choosing $\varepsilon=\log 2|\log t|^{-1}$. Note that $\varepsilon$ is admissible since it belongs to $(0,1)$ if $0<t<1 / 2$. With this choice of $\varepsilon$, (3.4) yields

$$
u(t) \leq C_{p}\|u\|_{W^{1, p}\left(B_{1}\right)}|\log t| \quad \text { for } \quad 0<t<1 / 2 .
$$

Using this and that $u$ is positive and decreasing, the desired logarithmic estimate (1.6) follows.

(c) Assume $n>p+4 p /(p-1)$ and $1 \leq q<q_{0}$, for $q_{0}$ defined as in (1.4). For $\varepsilon \in(0,1)$, let

$$
\alpha=1+\sqrt{\frac{n-1}{p-1}}-\varepsilon
$$

By (3.2), we have

$$
\begin{aligned}
u(t) & \leq \frac{C_{n, p}\|u\|_{W^{1, p}\left(B_{1}\right)}}{\varepsilon^{\frac{1}{p}}}\left(\int_{t}^{1} r^{\frac{p^{\prime}}{p}\left(-n+2 \sqrt{\frac{n-1}{p-1}}+3-2 \varepsilon\right)} d r\right)^{\frac{1}{p^{\prime}}} \\
& \leq C_{n, p}\|u\|_{W^{1, p}\left(B_{1}\right)} \frac{1}{\varepsilon^{\frac{1}{p}}} t^{-\frac{1}{p}\left(n-2 \sqrt{\frac{n-1}{p-1}}-p-2+2 \varepsilon\right)}
\end{aligned}
$$

for $0<t<1$, where we have used that $-\left(n-2 \sqrt{\frac{n-1}{p-1}}-p-2\right)<0$ since $n>p+4 p /(p-1)$ - see (1.5) .

Now, from (3.5) we obtain

$$
\int_{B_{1}} u^{q} d x \leq \frac{C_{n, p}^{q}\|u\|_{W^{1, p}\left(B_{1}\right)}^{q}}{\varepsilon^{\frac{q}{p}}} \int_{0}^{1} t^{-\left(n-2 \sqrt{\frac{n-1}{p-1}}-p-2+2 \varepsilon\right) \frac{q}{p}} t^{n-1} d t .
$$

If we set

$$
q=\frac{n p}{n-2 \sqrt{\frac{n-1}{p-1}}-p-2+3 \varepsilon}<q_{0}
$$

and $\varepsilon>0$ is small enough, the second integral of the previous inequality is finite. Hence, $u \in L^{q}\left(B_{1}\right)$ for every $1 \leq q<q_{0}$.

Finally, to prove the pointwise estimate (1.7) we consider (3.5) and proceed as in part (b). Now, we need to make $t^{-2 \varepsilon / p} / \varepsilon^{1 / p}$ small for given $t$. We take $\varepsilon=\log 2|\log t|^{-1}$, which belongs to $(0,1)$ if $0<t<1 / 2$. With this choice of $\varepsilon$, (3.5) leads to

$$
u(t) \leq C_{n, p}\|u\|_{W^{1, p}\left(B_{1}\right)} t^{-\frac{1}{p}\left(n-2 \sqrt{\frac{n-1}{p-1}}-p-2\right)}|\log t|^{\frac{1}{p}}
$$

for $t \in(0,1 / 2)$. Since $u$ is positive and decreasing, this leads to estimate (1.7) in all $B_{1}$. 
(d) Assume $g \geq 0$. We prove part (d1). Note that

$$
\partial_{r}\left(r^{n-1}\left|u_{r}\right|^{p-1}\right)=-\partial_{r}\left(r^{n-1}\left|u_{r}\right|^{p-2} u_{r}\right)=r^{n-1} g(u) \geq 0 \quad \text { in } B_{1}
$$

and hence $r^{n-1}\left|u_{r}\right|^{p-1}$ is a nonnegative and nondecreasing function of $r$. In particular

$$
\left\|r^{n-1}\left|u_{r}\right|^{p-1}\right\|_{L^{\infty}\left(B_{1}\right)} \leq\left|u_{r}(1)\right|^{p-1} .
$$

By (3.6) and since $u$ is bounded in $B_{1} \backslash B_{1 / 2}, r^{n-1}\left|u_{r}\right|^{p-1}$ is a $W^{1,1}(1 / 2,1)$ function of $r$ (indeed $\left.W^{1, \infty}\right)$. By the Sobolev embedding in one dimension, $r^{n-1}\left|u_{r}\right|^{p-1}$ is a continuous function up to $r=1$. Thus $\left|u_{r}(1)\right|$ in (3.7) is well defined and bounded. To control it, multiply equation (1.1) by $\varphi_{\varepsilon}(r):=\min \left\{1, \varepsilon^{-1}(1-r)\right\}$, where $\varepsilon \in(0,1)$. This yields

$$
\frac{1}{\varepsilon} \int_{B_{1} \backslash \bar{B}_{1-\varepsilon}}\left|u_{r}\right|^{p-1} d x=\int_{B_{1}} g(u) \varphi_{\varepsilon} d x .
$$

To fully justify (3.8), we need to multiply (1.1) by $\varphi_{\varepsilon} \zeta_{\delta}$, where $\zeta_{\delta}$ vanishes around 0 as in the proof of Lemma 2.3. Then we let $\delta \rightarrow 0$ and use that $u \in W^{1, p}\left(B_{1}\right)$ if $n \geq p$, and that $u \in C^{1}\left(\bar{B}_{1}\right)$ and $u_{r}(0)=0$ if $n<p$.

Now, letting $\varepsilon \rightarrow 0$ in (3.8) we obtain $\left|u_{r}(1)\right|^{p-1} \leq C_{n, p}\|g(u)\|_{L^{1}\left(B_{1}\right)}$. Thus, by (3.7) we deduce that

$$
\left\|r^{n-1}\left|u_{r}\right|^{p-1}\right\|_{L^{\infty}\left(B_{1}\right)} \leq C_{n, p}\|g(u)\|_{L^{1}\left(B_{1}\right)} .
$$

To control $\|\nabla u\|_{L^{p}\left(B_{1}\right)}$, assume first that $n<p$. Then,

$$
\begin{aligned}
\int_{0}^{1} r^{n-1}\left|u_{r}\right|^{p} d r & =\int_{0}^{1}\left(\left|u_{r}\right|^{p-1} r^{n-1}\right)^{\frac{p}{p-1}} r^{-\frac{n-1}{p-1}} d r \\
& \leq\left\|r^{n-1}\left|u_{r}\right|^{p-1}\right\|_{L^{\infty}\left(B_{1}\right)}^{\frac{p}{p-1}} \int_{0}^{1} r^{-\frac{n-1}{p-1}} d r .
\end{aligned}
$$

Since the last integral is finite, this and (3.9) lead to (1.8).

In case $n \geq p$, we use Lemma 2.3. We take $\alpha$ satisfying (2.7) and depending only on $n$ and $p$. The lemma gives that

$$
\begin{aligned}
& \int_{B_{1}} r^{-2 \alpha}\left|u_{r}\right|^{p} d x \\
& \quad \leq C_{n, p} \int_{B_{1}}\left|u_{r}\right|^{p} d x=C_{n, p} \int_{B_{r_{0}}}\left|u_{r}\right|^{p} d x+C_{n, p} \int_{B_{1} \backslash \bar{B}_{r_{0}}}\left|u_{r}\right|^{p} d x ;
\end{aligned}
$$

here we choose $r_{0} \in(0,1)$ satisfying $r_{0}^{-2 \alpha} \geq 2 C_{n, p}$. Since $C_{n, p} \int_{B_{r_{0}}}\left|u_{r}\right|^{p} d x \leq$ $(1 / 2) \int_{B_{r_{0}}} r^{-2 \alpha}\left|u_{r}\right|^{p} d x \leq(1 / 2) \int_{B_{1}} r^{-2 \alpha}\left|u_{r}\right|^{p} d x$, we can absorb the first term in the right-hand side of (3.11) into (3.10), and deduce that

$$
\int_{B_{1}}\left|u_{r}\right|^{p} d x \leq \int_{B_{1}} r^{-2 \alpha}\left|u_{r}\right|^{p} d x \leq 2 C_{n, p} \int_{B_{1} \backslash \bar{B}_{r_{0}}}\left|u_{r}\right|^{p} d x
$$


Note that $r_{0}$ depends only on $n$ and $p$, and thus, since $u$ is decreasing, $u\left(r_{0}\right)^{p-1} \leq$ $C_{n, p}\left\|u^{p-1}\right\|_{L^{1}\left(B_{r_{0}}\right)}$. Using this and (3.9), we have

$$
\begin{aligned}
& \int_{B_{1} \backslash \bar{B}_{r_{0}}}\left|u_{r}\right|^{p} d x=C_{n} \int_{r_{0}}^{1}\left|u_{r}\right|^{p} r^{n-1} d r \\
& \quad \leq C_{n}\left\|r^{n-1}\left|u_{r}\right|^{p-1}\right\|_{L^{\infty}\left(B_{1}\right)} \int_{r_{0}}^{1}-u_{r} d r \leq C_{n, p}\|g(u)\|_{L^{1}\left(B_{1}\right)}\left\|u^{p-1}\right\|_{L^{1}\left(B_{1}\right)}^{\frac{1}{p-1}}
\end{aligned}
$$

After using Young's inequality, this bound in $B_{1} \backslash \bar{B}_{r_{0}}$ and estimate (3.12) yield the desired bound (1.8) in all $B_{1}$.

Next, we prove $(\mathrm{d} 2)$. We consider first the case $n<p+4 p /(p-1)$. Observe that by part (a) we have that $u \in L^{\infty}\left(B_{1}\right)$. Hence, applying the regularity theory for the $p$-Laplacian (see [21]) it follows that $u \in C^{1, \beta}\left(\bar{B}_{1}\right)$ for some $0<\beta<1$. Moreover, by the $L^{\infty}$ estimate of part (a) above and estimate (1.8), there exists a constant depending on $n, p,\|u\|_{L^{1}\left(B_{1}\right)}$, and upper bounds in $g$ such that estimate (1.9) holds. This concludes the proof for $n<p+4 p /(p-1)$.

Assume $n \geq p+4 p /(p-1)$. We establish parts (d2) and (d3) at the same time. Observe that it is enough to prove our estimates in $B_{1 / 4}$. Indeed, since $u$ is decreasing, we have bounds for the supremum of $u$ in $B_{1} \backslash B_{1 / 5}$ in terms of $\|u\|_{L^{1}\left(B_{1}\right)}$. Thus by [21] we have that $u \in W^{1, q}\left(B_{1} \backslash \bar{B}_{1 / 5}\right)$ for all $q<\infty$. Moreover, $\|u\|_{W^{1, q}\left(B_{1} \backslash \bar{B}_{1 / 5}\right)} \leq C$, where $C$ is a constant depending on $n, p,\|u\|_{L^{1}\left(B_{1}\right)}$, and upper bounds in $g$.

We choose $\tilde{\rho} \in(1 / 4,1 / 2)$ such that

$$
0<-u_{r}(\tilde{\rho})=\frac{u(1 / 4)-u(1 / 2)}{1 / 4} \leq 4\|u\|_{L^{\infty}(1 / 4,1)} \leq C_{n, p}\|u\|_{W^{1, p}\left(B_{1}\right)},
$$

where in the last inequality we have used (2.1). On the other hand, from (2.2) we have

$$
\partial_{r}\left(\left|u_{r}\right|^{p-2} u_{r}\right)=-\frac{n-1}{r}\left|u_{r}\right|^{p-2} u_{r}-g(u) \leq-\frac{n-1}{r}\left|u_{r}\right|^{p-2} u_{r},
$$

for $r \in(0,1)$. We integrate (3.14) with respect to $r$, from $t$ to $\tilde{\rho}$, and use (3.13), to obtain

$$
\begin{aligned}
-\left|u_{r}(t)\right|^{p-2} u_{r}(t) & \leq-\left|u_{r}(\tilde{\rho})\right|^{p-2} u_{r}(\tilde{\rho})-(n-1) \int_{t}^{\tilde{\rho}} \frac{\left|u_{r}\right|^{p-2}}{r} u_{r} d r \\
& \leq C_{n, p}\|u\|_{W^{1, p}\left(B_{1}\right)}^{p-1}+(n-1) \int_{t}^{1 / 2} \frac{\left|u_{r}\right|^{p-1}}{r} d r
\end{aligned}
$$

for all $0<t<1 / 4$. Next, we estimate the last integral in (3.15) using Hölder 
inequality, to find

$$
\begin{gathered}
\int_{t}^{1 / 2} \frac{\left|u_{r}\right|^{p-1}}{r} d r=\int_{t}^{1 / 2}\left|u_{r}\right|^{p-1} r^{-(2 \alpha-n+1) / p^{\prime}} r^{\left(2 \alpha-n+1-p^{\prime}\right) / p^{\prime}} d r \\
\leq C_{n}\left(\int_{B_{1 / 2}}\left|u_{r}\right|^{p} r^{-2 \alpha} d x\right)^{\frac{1}{p^{\prime}}}\left(\int_{t}^{1 / 2} r^{p\left(2 \alpha-n+1-p^{\prime}\right) / p^{\prime}} d r\right)^{\frac{1}{p}}
\end{gathered}
$$

Let $\varepsilon \in(0,1)$ and

$$
\alpha=1+\sqrt{\frac{n-1}{p-1}}-\varepsilon
$$

Applying Lemma 2.3 in (3.16) and using that $p\left(2 \alpha-n+1-p^{\prime}\right)+p^{\prime}<0$ since $n \geq p+4 p /(p-1)$, we deduce from (3.15) and (3.16) the following estimate

$$
-u_{r}(t) \leq \frac{C_{n, p}}{\varepsilon^{1 / p}}\|u\|_{W^{1, p}\left(B_{1}\right)} t^{-\frac{1}{p}\left(n-2 \sqrt{\frac{n-1}{p-1}}-2+2 \varepsilon\right)},
$$

for $0<t<1 / 4$.

Now we use (3.17) to obtain, for $q \geq 1$,

$$
\int_{B_{1 / 4}}\left|u_{r}\right|^{q} d x \leq \frac{C_{n, p}}{\varepsilon^{q / p}}\left(\|u\|_{W^{1, p}\left(B_{1}\right)}\right)^{q} \int_{0}^{1} r^{n-1-\frac{q}{p}\left(n-2 \sqrt{\frac{n-1}{p-1}}-2+2 \varepsilon\right)} d r .
$$

If we set

$$
q=\frac{n p}{n-2 \sqrt{\frac{n-1}{p-1}}-2+3 \varepsilon}<q_{1}
$$

the second integral in (3.18) is finite for every $\varepsilon>0$. Hence, $u_{r} \in L^{q}\left(B_{1 / 4}\right)$ for every $q<q_{1}$. The estimate (1.9) follows, using in addition estimate (1.8).

Finally, the pointwise estimate (1.10) follows from (3.17) by choosing $\varepsilon=$ $\log 4|\log t|^{-1}$ for $0<t<1 / 4$.

\section{The extremal solution}

This section is devoted to prove Theorem 1.3. By estimate (1.8) of Theorem 1.2, now our task is to bound $u_{\lambda}^{p-1}$ and $f\left(u_{\lambda}\right)$ in $L^{1}\left(B_{1}\right)$ uniformly in $\lambda \in\left(0, \lambda^{*}\right)$. We prove this fact using the growth condition (1.13) on $f$ together with the radially decreasing property of the minimal solutions $u_{\lambda}$. These bounds lead to a control of $u_{\lambda}$ in $W^{1, p}\left(B_{1}\right)$ uniformly in $\lambda$, by estimate (1.8).

Proof of Theorem 1.3. We start noting that, for $\lambda \in\left(0, \lambda^{*}\right)$, the minimal solution $u_{\lambda} \in L^{\infty}\left(B_{1}\right)$ is radially decreasing. This follows from a general result of [9] on radially decreasing symmetry, which only requires $f$ to be positive in $(0, \infty)$ and locally Lipschitz in $[0, \infty)$ - see Corollary 1.1 in [9]. We can give, however, a 
simple proof in our situation of minimal solutions, as follows. First, the minimal solution $u_{\lambda}$ can be constructed by monotone iteration (see [7]), starting with $u^{0} \equiv 0$, by solving $-\Delta_{p} u^{k}=\lambda f\left(u^{k-1}(r)\right)$. Since the right-hand side is given and radial, the unique solution $u^{k}$ must be radial. Thus, the limit $u_{\lambda}$, as $k \rightarrow \infty$, is radial. Now, integrating our equation $-\partial_{r}\left(r^{n-1}\left|\partial_{r} u_{\lambda}\right|^{p-2} \partial_{r} u_{\lambda}\right)=r^{n-1} \lambda f\left(u_{\lambda}\right)>0$ in $r$, from 0 to $t \in(0,1)$, we get that $\partial_{r} u_{\lambda}(t)<0$.

For $\lambda \in\left(0, \lambda^{*}\right)$, let $\rho_{\lambda} \in(1 / 2,1)$ be such that

$$
\begin{aligned}
\left|\partial_{r} u_{\lambda}\left(\rho_{\lambda}\right)\right|^{p-1} & =\left(-\partial_{r} u_{\lambda}\left(\rho_{\lambda}\right)\right)^{p-1}=\left(\frac{u_{\lambda}(1 / 2)-u_{\lambda}(1)}{1 / 2}\right)^{p-1} \\
& =\left(2 u_{\lambda}(1 / 2)\right)^{p-1} \leq C_{n, p}\left\|u_{\lambda}^{p-1}\right\|_{L^{1}\left(B_{1 / 2}\right)}
\end{aligned}
$$

where we have used that $u_{\lambda}$ is radially decreasing. Since $r^{n-1}\left|\partial_{r} u_{\lambda}\right|^{p-1}$ is increasing in $r \in(0,1)$ by (3.6), the previous estimate yields

$$
\left\|r^{n-1}\left|\partial_{r} u_{\lambda}\right|^{p-1}\right\|_{L^{\infty}\left(B_{1 / 2}\right)} \leq \rho_{\lambda}^{n-1}\left|\partial_{r} u_{\lambda}\left(\rho_{\lambda}\right)\right|^{p-1} \leq C_{n, p}\left\|u_{\lambda}^{p-1}\right\|_{L^{1}\left(B_{1 / 2}\right)} .
$$

Multiply equation $\left(1.12_{\lambda, p}\right)$ by $\psi(r)=\min \left\{1,4(1 / 2-r)^{+}\right\}$, to obtain

$$
\begin{aligned}
\left\|\lambda f\left(u_{\lambda}\right)\right\|_{L^{1}\left(B_{1 / 4}\right)} & \leq C_{n, p} \int_{1 / 4}^{1 / 2} r^{n-1}\left|\partial_{r} u_{\lambda}\right|^{p-1} d r \\
& \leq C_{n, p}\left\|u_{\lambda}^{p-1}\right\|_{L^{1}\left(B_{1 / 2}\right)},
\end{aligned}
$$

where we have used (4.1). Note that here we can test the equation with $\psi$, a function which does not vanish around the origin, since we know that $u_{\lambda}$ is an energy solution in all of $B_{1}$.

Next, we use assumption (1.13) on $f$ to ensure, given any $\delta>0$, that $\lambda f(t) \geq$ $\frac{1}{\delta} t^{p-1}-C_{\delta}$ for all $t>0$ and $\lambda \in\left(\lambda^{*} / 2, \lambda^{*}\right)$, where $C_{\delta}$ does not depend on $\lambda$. This combined with (4.2) leads to

$$
\left\|u_{\lambda}^{p-1}\right\|_{L^{1}\left(B_{1 / 4}\right)} \leq C_{n, p} \delta\left\|u_{\lambda}^{p-1}\right\|_{L^{1}\left(B_{1 / 2}\right)}+C_{\delta}
$$

Now, since $u_{\lambda}$ is decreasing in $r$, we have

$$
\left\|u_{\lambda}^{p-1}\right\|_{L^{1}\left(B_{1 / 2} \backslash \bar{B}_{1 / 4}\right)} \leq C_{n, p} u_{\lambda}^{p-1}(1 / 4) \leq C_{n, p}\left\|u_{\lambda}^{p-1}\right\|_{L^{1}\left(B_{1 / 4}\right)} .
$$

This combined with (4.3) yields, after taking $\delta$ small enough, $\left\|u_{\lambda}^{p-1}\right\|_{L^{1}\left(B_{1 / 4}\right)} \leq C$ for a constant $C$ independent of $\lambda$. This bound and the argument used in (4.4) done now on $B_{1} \backslash \bar{B}_{1 / 4}$ leads to a uniform in $\lambda$ bound for $\left\|u_{\lambda}^{p-1}\right\|_{L^{1}\left(B_{1}\right)}$.

The previous bound gives also a control for $\left\|f\left(u_{\lambda}\right)\right\|_{L^{1}\left(B_{1 / 4}\right)}$, by (4.2). Since $f$ is increasing, $f\left(u_{\lambda}\right)$ is decreasing in $r$ and the argument used above allows to control $\left\|f\left(u_{\lambda}\right)\right\|_{L^{1}\left(B_{1}\right)}$ uniformly in $\lambda$. Thus,

$$
\left\|u_{\lambda}^{p-1}\right\|_{L^{1}\left(B_{1}\right)}+\left\|f\left(u_{\lambda}\right)\right\|_{L^{1}\left(B_{1}\right)} \leq C
$$


for some constant $C$ independent of $\lambda \in\left(0, \lambda^{*}\right)$. By estimate (1.8) of Theorem 1.2, we deduce a bound for $\left\|\nabla u_{\lambda}\right\|_{L^{p}\left(B_{1}\right)}$ independent of $\lambda$. Since $\left.u_{\lambda}\right|_{\partial B_{1}} \equiv 0$, this yields a bound for $\left\|u_{\lambda}\right\|_{W^{1, p}\left(B_{1}\right)}$.

Thus, we have that there exists $v \in W_{0}^{1, p}\left(B_{1}\right)$ and some subsequence still denoted by $u_{\lambda}$ such that, as $\lambda \rightarrow \lambda^{*}, u_{\lambda} \rightarrow v$ weakly in $W_{0}^{1, p}\left(B_{1}\right), u_{\lambda} \rightarrow v$ strongly in $L^{p}\left(B_{1}\right)$, and $u_{\lambda} \rightarrow v$ a.e. in $B_{1}$. Since $u_{\lambda}$ is increasing in $\lambda$, every sequence $u_{\lambda}(r)$ tends as $\lambda \rightarrow \lambda^{*}$ to $u^{*}(r)$, by the definition (1.14) of $u^{*}$. Hence, $v=u^{*} \in W_{0}^{1, p}\left(B_{1}\right)$.

Next, we show that $u^{*}$ is an energy solution of $\left(1.12_{\lambda^{*}, p}\right)$. We want to pass to the limit in

$$
\int_{B_{1}}\left|\nabla u_{\lambda}\right|^{p-2} \nabla u_{\lambda} \cdot \nabla \varphi d x=\int_{B_{1}} \lambda f\left(u_{\lambda}\right) \varphi d x \quad \text { for } \varphi \in C_{c}^{1}\left(B_{1}\right) .
$$

Since $f\left(u_{\lambda}\right)$ increases to $f\left(u^{*}\right)$ and $\left\|f\left(u_{\lambda}\right)\right\|_{L^{1}\left(B_{1}\right)}$ is uniformly bounded in $\lambda$, the monotone convergence theorem gives that $f\left(u^{*}\right) \in L^{1}\left(B_{1}\right)$ and that the limit as $\lambda \rightarrow \lambda^{*}$ of the right-hand side in (4.5) is

$$
\lambda^{*} \int_{B_{1}} f\left(u^{*}\right) \varphi d x
$$

To pass to the limit in the left-hand side of (4.5), note first that we may assume that $\varphi$ is radial - simply by integrating first with respect to the "angles" $\theta \in \partial B_{1}$ in (4.5). Now, the left-hand side of (4.5), up to a multiplicative constant, reads

$$
\int_{0}^{1} r^{n-1}\left|\partial_{r} u_{\lambda}\right|^{p-1}\left(-\partial_{r} \varphi\right)(r) d r
$$

Using (3.6) and that the $L^{1}(0,1)$ norm of $r^{n-1} f\left(u_{\lambda}(r)\right)$ is bounded uniformly in $\lambda$, we deduce that $r^{n-1}\left|\partial_{r} u_{\lambda}\right|^{p-1}$ is bounded in $W^{1,1}(0,1)$ 一 the Sobolev space in one dimension - uniformly in $\lambda \in\left(0, \lambda^{*}\right)$. Since this space is compactly embedded in $L^{1}(0,1)$, we conclude that $r^{n-1}\left|\partial_{r} u_{\lambda}\right|^{p-1}$ converges strongly in $L^{1}(0,1)$ to $r^{n-1}\left|\partial_{r} u^{*}\right|^{p-1}$. Hence, we can pass to the limit in (4.6) and conclude

$$
\int_{B_{1}}\left|\nabla u^{*}\right|^{p-2} \nabla u^{*} \cdot \nabla \varphi d x=\lambda^{*} \int_{B_{1}} f\left(u^{*}\right) \varphi d x,
$$

for every $\varphi \in C_{c}^{1}\left(B_{1}\right)$, proving the claim.

Clearly, $u^{*}$ is a radially symmetric solution and it is nonincreasing. Hence, by regularity, $u^{*} \in C^{1}\left(\bar{B}_{1} \backslash\{0\}\right)$ since $u^{*} \in L^{\infty}\left(B_{1} \backslash\{0\}\right)$. Therefore, using that $u^{*} \in W_{0}^{1, p}\left(B_{1}\right)$ and $f>0$, the argument at the end of the first paragraph of this proof —or Hopf Lemma (see [24] or Lemma A.3 in [25]) -, lead to $u_{r}^{*}(t)<0$ for all $t \in(0,1)$. That is, $u^{*}$ is radially decreasing.

At this point we are able to prove that $u^{*}$ is semi-stable in the sense of Definition 1.1. That is,

$$
\int_{B_{1}} \lambda^{*} f^{\prime}\left(u^{*}\right) \xi^{2} d x \leq \int_{B_{1}}(p-1)\left|u_{r}^{*}\right|^{p-2}\left|\xi_{r}\right|^{2} d x
$$


for every radially symmetric function $\xi \in C_{c}^{1}\left(B_{1} \backslash\{0\}\right)$. This follows by passing to the limit as $\lambda \rightarrow \lambda^{*}$ in the corresponding semi-stability property for the minimal solutions $u_{\lambda}$. On the left-hand sides, we simply, use Fatou's lemma (recall that $f^{\prime} \geq 0$ ). On the right-hand side, we use that $\xi$ has compact support in $B_{1} \backslash\{0\}$, and that in such compact set we have $C^{2, \beta}$ estimates for $u_{\lambda}$ uniformly in $\lambda \in$ $\left(0, \lambda^{*}\right)$. This holds since $\left|\partial_{r} u_{\lambda}^{*}\right|>0$ in such compact set, and the same is true for $\left|\partial_{r} u_{\lambda}\right|$ uniformly in $\lambda \in\left(0, \lambda^{*}\right)$, and thus we deal with uniformly elliptic equations. Thus $\left|\partial_{r} u_{\lambda}\right|$ converges uniformly to $\left|u_{r}^{*}\right|$, and the same is true for the quantities $\left|\partial_{r} u_{\lambda}\right|^{p-2}$, since $\left|u_{r}^{*}\right|>0$ - note that the exponent $p-2$ could be negative.

Finally, the regularity statements in the theorem follow as a consequence of Theorem 1.2 ,

\section{Acknowledgments}

The authors were supported by the Ministerio de Educación y Ciencia (Spain), grant MTM2005-07660-C02-01. The authors are partially sponsored by the European Science Foundation (ESF) PESC Programme "Global".

The second author is also supported by the E.U. RTN program "MULTIMAT" MRTN-CT-2004-505226.

The third author was also supported by CMUC/FCT (Coimbra, Portugal).

\section{References}

[1] H. Brézis, J.L. Vázquez, Blow-up solutions of some nonlinear elliptic problems, Rev. Mat. Univ. Compl. Madrid 10 (1997), 443-469.

[2] X. Cabré, Boundedness of minimizers of semilinear elliptic problems up to dimension four, in preparation.

[3] X. Cabré, Extremal solutions and instantaneous complete blow-up for elliptic and parabolic problems, Contemporary Mathematics 446, American Math. Soc. 2007, in: Perspectives in Nonlinear Partial Differential Equations: In honor of Haïm Brezis, pp. 159-174.

[4] X. Cabré, A. Capella, On the stability of radial solutions of semilinear elliptic equations in all of $\mathbb{R}^{n}$, C. R. Math. Acad. Sci. Paris 338 (2004), 769-774.

[5] X. Cabré, A. Capella, Regularity of radial minimizers and extremal solutions of semilinear elliptic equations, J. Funct. Anal. 238 (2006), 709-733.

[6] X. Cabré, A. Capella, Regularity of minimizers for three elliptic problems: minimal cones, harmonic maps, and semilinear equations, Pure and Applied Math Quarterly 3 (2007), 801-825. 
[7] X. Cabré, M. Sanchón, Semi-stable and extremal solutions of reaction equations involving the p-Laplacian, Comm. Pure Appl. Anal. 6 (2007), 43-67.

[8] M.G. Crandall, P.H. Rabinowitz, Some continuation and variational methods for positive solutions of nonlinear elliptic eigenvalue problems, Arch. Ration. Mech. Anal. 58 (1975), 207-218.

[9] L. Damascelli, B. Sciunzi, Regularity, monotonicity and symmetry of positive solutions of m-Laplace equations, J. Differential Equations 206 (2004), 483515.

[10] J. Dávila, Singular solutions of semi-linear elliptic problems, preprint.

[11] J. Dávila, L. Dupaigne, Perturbing singular solutions of the Gelfand problem, Commun. Contemp. Math. 9 (2007), 639-680.

[12] J. Dávila, L. Dupaigne, I. Guerra, M. Montenegro, Stable solutions for the bilaplacian with exponential nonlinearity, SIAM J. Math. Anal. 39 (2007), 565-592 (electronic).

[13] J. Dávila, L. Dupaigne, M. Montenegro, The extremal solution of a boundary reaction problem, preprint.

[14] S. Eidelman, Y. Eidelman, On regularity of the extremal solution of the Dirichlet problem for some semilinear elliptic equations of the second order, Houston J. Math. 31 (2005), 957-960.

[15] P. Esposito, Compactness of a nonlinear eigenvalue problem with singular nonlinearity, preprint.

[16] A. Ferrero, On the solutions of quasilinear elliptic equations with a polynomial-type reaction term, Adv. Differential Equations 9 (2004), 1201-1234.

[17] J. García-Azorero, I. Peral, On an Emden-Fowler type equation, Nonlinear Anal. 18 (1992), 1085-1097.

[18] J. García-Azorero, I. Peral, J.P. Puel, Quasilinear problems with exponential growth in the reaction term, Nonlinear Anal. 22 (1994), 481-498.

[19] N. Ghoussoub, Y. Guo, On the partial differential equations of electrostatic MEMS devices: stationary case, SIAM J. Math. Anal. 38 (2007), 1423-1449.

[20] D.D. Joseph, T.S. Lundgren, Quasilinear Dirichlet problems driven by positive sources, Arch. Ration. Mech. Anal. 49 (1973), 241-269.

[21] G.M. Lieberman, Boundary regularity for solutions of degenerate elliptic equations, Nonlinear Anal. 11 (1988), 1203-1219. 
[22] F. Mignot, J.P. Puel, Sur une classe de problèmes non linéaires avec nonlinéarité positive, croissante, convexe, Comm. Partial Differential Equations 5 (1980), 791-836.

[23] G. Nedev, Regularity of the extremal solution of semilinear elliptic equations, C. R. Acad. Sci. Paris Sér. I Math. 330 (2000), 997-1002.

[24] I. Peral, Multiplicity of solutions for the p-Laplacian, International Center for Theoretical Physics Lecture Notes, Trieste, 1997.

[25] S. Sakaguchi, Concavity properties of solutions to some degenerate quasilinear elliptic Dirichlet problems, Ann. Scuola Norm. Sup. Pisa Cl. Sci. 14 (1987), 403-421.

[26] M. Sanchón, Boundedness of the extremal solution of some p-Laplacian problems, Nonlinear Anal. 67 (2007), 281-294.

[27] M. Sanchón, Existence and regularity of the extremal solution of some nonlinear elliptic problems related to the p-Laplacian, Potential Anal. 27 (2007), $217-224$.

[28] S. Villegas, Asymptotic behavior of stable radial solutions of semilinear elliptic equations in $\mathbb{R}^{N}$, to appear in J. Math. Pures et Appliquees.

[29] S. Villegas, Sharp estimates for semi-stable radial solutions of semilinear elliptic equations, preprint. 